\title{
P02.166. Group yoga intervention leads to improved balance and balance self-efficacy after stroke
}

\author{
A Schmid ${ }^{1 *}$, M Van Puymbroeck ${ }^{2}$, K Miller $^{1}$, N Schalk ${ }^{3}$ \\ From International Research Congress on Integrative Medicine and Health 2012 \\ Portland, Oregon, USA. 15-18 May 2012
}

\section{Purpose}

Evaluate the impact of an 8-week group yoga intervention on balance, balance self-efficacy, and falls-efficacy in individuals with chronic stroke.

\section{Methods}

This is a prospective pilot study of a modified yoga intervention. All study participants: had chronic stroke ( $>9$ months); completed all rehabilitation after stroke; were able to stand but self-reported some residual disability related to walking, balance, or functional loss after stroke; and scored $>4$ out of 6 on the Short Mini Mental Status Exam. Forty-seven individuals with stroke were recruited and randomized 3:1 to yoga or waitlist control. The yoga group completed one hour yoga sessions twice a week for 8 weeks. Yoga was taught by a certified yoga therapist and included modified physical postures, yoga breathing, bilateral movements, and concluded with relaxation while seated, standing, and supine. Assessments before and after the 8 weeks included: Berg Balance Score (balance), Activities Balance Confidence Scale (ABC, balance self-efficacy), and Falls-Efficacy Scale (falls-efficacy). We compared groups with a t-test/Mann Whitney. We used paired $t$-tests to compare baseline and 8-week data.

\section{Results}

The average age of participants completing the study was 64; $76 \%$ were male; and $63 \%$ were white. There were no differences in demographics or outcomes between the yoga and control groups. There were no improvements in the waitlist control group. In the yoga group $(n=29)$, significant improvements were found after the 8-week intervention in balance (Berg $40.7 \pm 12.1$ vs $47 \pm 9.6$, $\mathrm{p}<0.001)$ and balance self-efficacy ( $A B C 61.25 \pm 21.8$ vs $67.2 \pm 23.1, \mathrm{p}=0.035)$. Falls-efficacy did not improve $(\mathrm{p}=0.164)$.

\section{Conclusion}

Our findings suggest an 8-week yoga intervention impacts balance and balance self-efficacy for people with chronic stroke. Yoga activities may have improved neuromuscular control, allowing for strength improvements in affected limbs/side or areas of disuse, thereby improving balance. Continued testing with a larger sample is warranted to determine the impact of yoga on balance and self-efficacy.

\section{Author details}

${ }^{1}$ Roudebush VAMC and Indiana University, Indianapolis, USA. ${ }^{2}$ Indiana University, Bloomington, USA. ${ }^{3}$ Heartland Yoga, Indianapolis, USA.

Published: 12 June 2012

doi:10.1186/1472-6882-12-S1-P222

Cite this article as: Schmid et al.: P02.166. Group yoga intervention leads to improved balance and balance self-efficacy after stroke. BMC Complementary and Alternative Medicine 2012 12(Suppl 1):P222.

${ }^{1}$ Roudebush VAMC and Indiana University, Indianapolis, USA

Full list of author information is available at the end of the article

(c) 2012 Schmid et al; licensee BioMed Central Ltd. This is an Open Access article distributed under the terms of the Creative Commons 